\title{
La marginalidad, la vulnerabilidad y su implicancia en los problemas sociales y estructurales de una sociedad
}

\author{
Mario Phillip Roncal Zolezzi \\ Universidad Antonio Ruiz de Montoya
}

\section{Resumen}

El artículo muestra la falta de capacidades del Estado para entender, analizar, planificar y resolver problemas endémicos que generan la pobreza y cómo ellos nos llevan a situaciones de marginación, vulnerabilidad e informalidad. La relación que tiene el efecto que conlleva el no actuar apropiadamente para lograr un cambio en esta situación permite que personas que son parte de nuestra sociedad no puedan lograr tomar decisiones propias sobre su destino y dependan de las políticas de apoyo que el Estado determine y que siempre son limitadas, como sus vidas y anhelos.

Palabras clave: vulnerabilidad, pobreza, informalidad, precariedad, marginalidad, educación, distanciamiento social.

\begin{abstract}
The article shows the lack of capacities of the State to understand, analyze, plan, and solve endemic problems that generate poverty and how they lead us to situations of marginalization, vulnerability and informality. The relationship that has the effect of not acting appropriately to achieve a change in this situation, allows people who are part of our society to not be able to make their own decisions about their destiny and depend on the support policies that the State determines and that are always limited, like their lives and desires.
\end{abstract}

Keywords: vulnerability, poverty, informality, precariousness, marginality, education, social distancing. 


\section{Introducción}

La situación de precariedad y pobreza, la informalidad, el desempleo, la falta de servicios básicos y servicios públicos podrían darnos una idea del significado del distanciamiento social. La pobreza margina las decisiones de las personas y las lleva a un estado de subsistencia. La informalidad podría llamarse una pandemia del Estado, debido a las consecuencias que genera el permitirla y no buscar soluciones para reducirla.

Hernando de Soto, en su libro El misterio del capital, habla sobre el problema de la propiedad privada y de las poblaciones migrantes que invadieron vastas zonas aledańas a las ciudades y se asentaron inicialmente en precarias viviendas. Estos lugares fueron denominados pueblos jóvenes o asentamientos humanos, donde sin articulación urbana hoy pasan a ser las zonas más pobladas de las ciudades. Sin embargo, los pobladores no gozan de la propiedad de estas, por no tener título de propiedad del terreno en que viven. El Estado ha permitido por años estas invasiones; lo que no ha hecho es formalizar su propiedad.

Un problema derivado de esta migración y el aumento de la densidad poblacional es la falta de servicios públicos (educación, salud, luz, agua, seguridad, etc.), que hace que el Estado no pueda ofrecer los adecuados recursos para lograr sostener a estos grupos humanos que quedan marginados de la sociedad. Matos (1984) nos habla de dos Perúes paralelos refiriéndose a la migración: "Por un lado el Perú Oficial de las instituciones del Estado, los partidos, la banca y las empresas, los sindicatos, las universidades y colegios, las Fuerzas Armadas y la Iglesia; de los tribunales, la burocracia y el papel sellado; de la cultura exocéntrica. Y, por el otro, el Perú Marginado: plural y multiforme; del campesinado y la masa urbana, de las asociaciones de vecinos, los cabildos tradicionales, las rondas y los varayoc; de los talleres clandestinos, los ambulantes y las economías de trueque, de reciprocidad y de mera subsistencia; de los cultos de los cerros, la espera de Inkarrí y la devoción a las santas y beatas no canonizadas; el Perú que conserva, adapta y fusiona innumerables tradiciones locales y 
regionales; bilingüe, analfabeto y a veces monolingüe quechua, aymara o amazónico. Este contraste, gestado desde los primeros tiempos de la Colonia, se prolonga hasta avanzado el Perú Republicano".

Matos (1984) nos indica que "las invasiones de tierras y de predios urbanos que caracterizaron la década del cincuenta se mostraron en el curso de los ańos como precursoras de más importantes y poderosas invasiones: la de los predios urbanos de la cultura oficial, por la cultura andina y la de los ámbitos de la economía, la política, la educación, el mundo jurídico y la religión por los nuevos estilos impuestos por las masas en constante desborde y expansión". Sobre las invasiones de terrenos por parte de las poblaciones migrantes, Foschiatti (2007) afirma lo siguiente: "[...] con su temprano proceso de urbanización, América Latina ha ocasionado el progresivo avance de la pobreza hacia las ciudades, provocando el fenómeno denominado urbanización de la pobreza, convirtiendo a las mismas en el ambiente para su difusión y por tanto en sectores que requieren de políticas públicas para combatirla”.

Asimismo, Sandoval (2014) indica que la migración de las poblaciones hacia las ciudades, el desempleo, la complementariedad activa entre la economía informal y formal, el exceso de normas y leyes, la laxitud en el cumplimiento de estas, razones políticas y de corrupción, en parte son características que podrían definir las causas de la informalidad, debido a la necesidad de subsistencia.

A través de un estudio económico publicado en la revista del Banco Central de Reserva del Perú, Loayza (2008) afirma lo siguiente:

la informalidad es la forma distorsionada con la que una economía excesivamente reglamentada responde tanto a los choques que enfrenta, como a su potencial de crecimiento. Se trata de una respuesta distorsionada, porque la informalidad supone una asignación de recursos deficiente que conlleva la pérdida, por lo menos parcial, de las ventajas que ofrece la legalidad: la protección policial y judicial, el acceso al crédito formal, y la capacidad de participar en los mercados internacionales. 
Llámese marginalidad, informalidad, vulnerabilidad o distanciamiento social, podemos analizar que la falta de inclusión de las poblaciones que viven en los entornos de las ciudades y la dejadez de las autoridades de gobierno ha generado una falta de planificación y desarrollo urbano, ha permitido que poblaciones migrantes se establezcan en lugares poco favorecidos de la ciudad, por lo que no permiten el fácil acceso a las instalaciones de agua y luz.

Definir los parámetros que marcan la marginalidad ha generado un debate muy grande en la comunidad científica a la hora de analizar la realidad social, especialmente en las situaciones de vulnerabilidad, debido a que se utilizan diversos enfoques y técnicas (indicadores de calidad de vida, indicadores de exclusión social, de contexto, autosuficiencia, equidad, salud, entre otros), (Navarro y Larrubia, 2006).

Existe una encrucijada entre el modernismo, llámese globalización, y la falta de instrumentos tecnológicos, capacidad profesional, recursos con que cuentan las poblaciones vulnerables; esta desigualdad en educación, integración, comunicaciones y trabajo limita sus oportunidades.

\section{Desempleo, educación y vulnerabilidad}

Si analizamos cifras teniendo en cuenta el factor educación y su correlato con el empleo, vemos que en la ciudad de Lima con 9674755 habitantes y que representa el 29,7 \% de la población total del Perú, ha habido una disminución significativa en la población económicamente activa del 37,2 \% (teniendo en cuenta el período junio-julio-agosto de 2019 y junio-julio-agosto de 2020); de esta última cifra (37,2 \%), la población que solo cuenta con educación escolar representa el $65 \%$. Analizando este último porcentaje, nos da una cifra de 2326,8 millones de personas (Cuadro N¹ - INEI 2020), con lo cual podemos observar que la población que solo cuenta con educación escolar se va a ver más afectada respecto de la pérdida de empleo que la más instruida.

Carrillo et al. (2019), en un estudio realizado para el Instituto de Estudios Peruanos, indican que la oferta de colegios públicos y de bajo costo se encuentran en los distritos de Lima de menor nivel 
socioeconómico. Asimismo, se evidencia que los estudiantes de familias con menores ingresos van a tener menores oportunidades de aprendizaje y, por lo tanto, resultados educativos más bajos que el resto de la población.

\section{Cuadro $\mathbf{N}^{\circ} 1$}

Lima Metropolitana: Población Económicamente Activa, según sexo, edad y nivel de educación alcanzado

Trimestre móvil: Jun-jul-ago 2019 y Jun-jul-ago 2020

(Miles de personas, variación absoluta y porcentual)

\begin{tabular}{|c|c|c|c|c|}
\hline \multirow[b]{2}{*}{ Caracteristicas } & \multirow{2}{*}{$\begin{array}{l}\text { Jun-jul- } \\
\text { ago } 2019\end{array}$} & \multirow{2}{*}{$\begin{array}{c}\text { Jun-jul- } \\
\text { ago } 2020\end{array}$} & \multicolumn{2}{|c|}{ Variación } \\
\hline & & & $\begin{array}{c}\text { Absoluta } \\
\text { (miles) }\end{array}$ & $\begin{array}{l}\text { Porcentual } \\
\quad \text { (96) }\end{array}$ \\
\hline Total & 5237,3 & 4277,1 & (-) 960,2 & $(-) 18,3$ \\
\hline \multicolumn{5}{|l|}{ Sexo } \\
\hline Hombre & 2810,0 & 2389,5 & (-) 420,5 & (-) 15,0 \\
\hline Mujer & 2427,2 & 1887,5 & (-) 539,7 & (-) 22,2 \\
\hline \multicolumn{5}{|l|}{ Grupos de edad } \\
\hline De 14 a 24 años & 951,2 & 683,2 & (-) 268,0 & (-) 28,2 \\
\hline De 25 a 44 años & 2739,7 & 2385,1 & (-) 354,6 & $(-) 12,9$ \\
\hline De 45y más años & 1546,3 & 1208,8 & $(-) 337,5$ & (-) 21,8 \\
\hline \multicolumn{5}{|l|}{ Nivel de Educación } \\
\hline Primaria $1 /$ & 389,1 & 214,0 & $(-) 175,1$ & (-) 45,0 \\
\hline Secundaria & 2482,8 & 1913,7 & (-) 569,1 & (-) 22,9 \\
\hline Superior no universitaria & 950,1 & 856,8 & $(-) \quad 93,3$ & $(-) \quad 9,8$ \\
\hline Superior universitaria & 1415,3 & 856,9 & (-) 122,7 & $(-) \quad 8,7$ \\
\hline
\end{tabular}

1/ Incluye inicial y sin nivel.

Fuente: Instituto Nacional de Estadística e informática -Encuesta Permanente de Empleo

Cuenca y Urrutia (2019) realizan un análisis sobre las brechas que existen en la gestión educativa en el Perú. En el siguiente cuadro (Cuadro $\mathrm{N}^{\circ}$ 2), analizan el coeficiente de Gini de los años de educación para las personas de hogares pobres y no pobres que dejaron de educarse entre el 2004 y el 2016. Se indica que las personas pobres en el 2016 
presentan las mismas características educativas que tenían en el 2004, lo que quiere decir que no ha habido un incremento en su nivel educativo. Por el contrario, las personas con una mejor situación económica han sido favorecidas por un incremento sostenido en el nivel educativo.

\section{Cuadro $\mathrm{N}^{\circ} 2$}

\section{Coeficiente de Gini y promedio de los años de educación según pobreza}
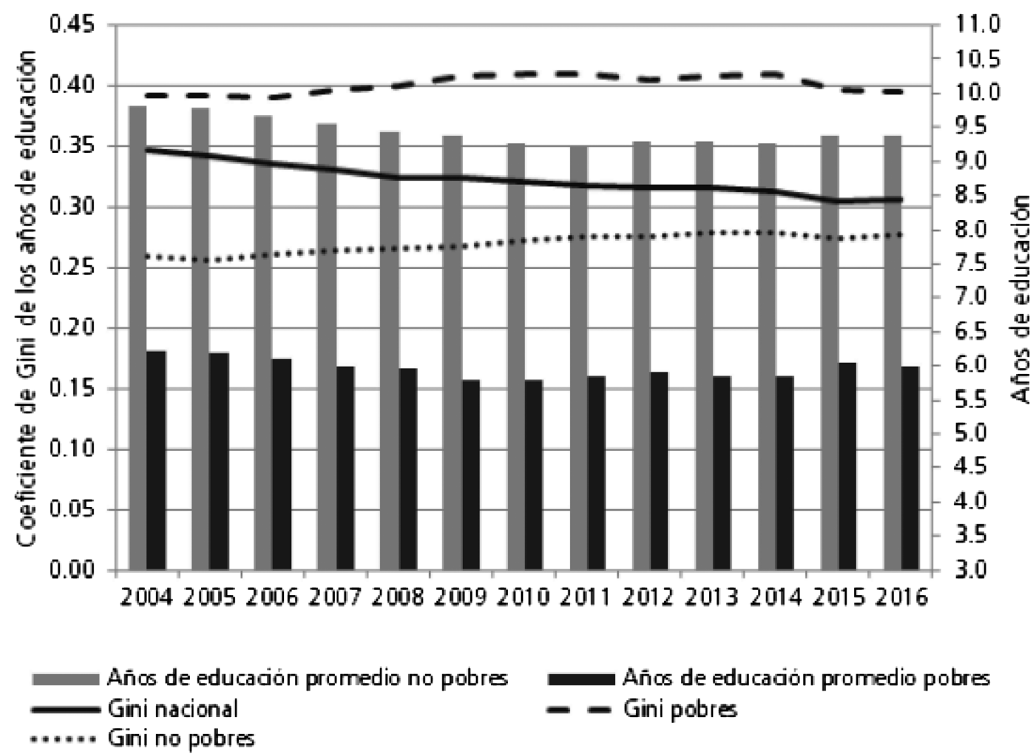

Fuente: Elaboración Cuenca y Urrutia, con base en la Encuesta Nacional de Hogares (INEI, 2018).

De los párrafos anteriores, se desprende que la base de una buena educación determina las posibilidades de las personas a definir su propio destino, lo que es esquivo a las poblaciones de menos recursos. Castro y Cano (2013), relacionando los factores de riesgo que generan la pobreza y vulnerabilidad en el desarrollo educativo, indican que los niños y las niñas se encuentran sujetos a la posibilidad de un 
crecimiento y un desarrollo futuro inadecuados. Recalca que las familias económicamente desfavorecidas presentan factores que perjudican seriamente su labor frente al apoyo y responsabilidad de los padres en las labores educativas de sus hijos; características de ello son sus niveles educativos, falta de trabajo o ingresos mínimos salariales, la extrema pobreza, ausencia de los padres en el hogar, entre otros.

La problemática de la pobreza basada en la falta de educación está relacionada directamente con el grado de instrucción de la población; esta determina el nivel económico de la persona, la posibilidad del sustento familiar y su desarrollo en la sociedad. Pasar la línea de pobreza está relacionada con las capacidades técnicas de las personas para poder incrementar su nivel remunerativo (Castro et al., 2017).

Existe una falta motivación que está generada por los factores de pobreza en las relaciones de convivencia y la ruptura familiar, y está ligada a la participación familiar y al esfuerzo frente a las responsabilidades del hogar. Intervienen en este contexto la cultura, el rol del Estado, la sociedad frente a la realidad de los padres, las madres y los niños en estado de vulnerabilidad. Sin embargo, uno de los problemas más graves de vulnerabilidad se presenta en los niños en el largo plazo, debido a que internalizan los problemas del hogar en todas sus dimensiones, además de convivir con una mala alimentación, salud física y mental (Stock et al., 2014).

Hemos analizado cifras que dejan un resultado muy complicado y que nos permiten ver que la situación de la pobreza en el Perú avanza con un aliado que es la precaria educación que reciben nuestros estudiantes de niveles socioeconómicos bajos; las cifras recogidas del INEI nos muestran un panorama poco alentador y subrayan lo indicado en párrafos anteriores. En la ciudad de Lima, el 46,0 \% de la población en edad de trabajar ( 3 millones 643 mil 100 personas), pertenece a la población económicamente no activa (NO PEA); en comparación con el análisis del trimestre similar del año anterior, aumentó en 41,3 \% (1 millón 64 mil 100 personas). (Ver Cuadro N³).

Esta sensación de vulnerabilidad, la falta de recursos y un futuro no muy claro producen en las poblaciones de extrema pobreza y sin apoyo de las instituciones tutelares, que "tiendan a la exclusión, la 
marginalidad de los grupos más pobres conforman el panorama social de las familias pobres, con educación incompleta, con dificultades de acceder a una vivienda digna, a los servicios elementales, a un empleo estable, lo que conlleva a la delincuencia, a la violencia, ejes de una problemática que se instala cada vez con mayor fuerza en las áreas menos desarrolladas, con falta de políticas sociales que orienten hacia la equidad, el pleno empleo y la organización de la sociedad" (Foschatti, 2007).

\section{Cuadro $\mathrm{N}^{\circ} 3$}

Lima Metropolitana: Población en edad de trabajar, según condición de actividad Trimestre móvil: Jun-jul-ago 2019 y Jun-jul-ago 2020

(Miles de personas, variación absoluta y porcentual)

\begin{tabular}{|c|c|c|c|c|}
\hline \multirow[b]{2}{*}{ Condición de actividad } & \multirow{2}{*}{$\begin{array}{l}\text { Jun-jul- } \\
\text { ago } 2019\end{array}$} & \multirow{2}{*}{$\begin{array}{l}\text { Jun-jul- } \\
\text { ago } 2020\end{array}$} & \multicolumn{2}{|c|}{ Variación } \\
\hline & & & $\begin{array}{l}\text { Absoluta } \\
\text { (miles) }\end{array}$ & $\begin{array}{l}\text { Porcen- } \\
\text { tual (96) }\end{array}$ \\
\hline & & & (Miles) & $(\%)$ \\
\hline $\begin{array}{l}\text { Total de población en edad } \\
\text { trabajar (PET) }\end{array}$ & 7816,3 & 7920,1 & 103,8 & 1,3 \\
\hline $\begin{array}{l}\text { Población económicamente } \\
\text { activa (PEA) }\end{array}$ & 5237,3 & 4277,1 & 960,2 & $-18,3$ \\
\hline Ocupada & 4932,1 & 3611,3 & (-) 1320,8 & $-26,8$ \\
\hline Desocupada & 305,2 & 665,8 & 360,6 & 118.2 \\
\hline $\begin{array}{l}\text { Población económicamente } \\
\text { no activa (NO PEA) }\end{array}$ & 2579,0 & 3643,1 & 1064,1 & 41,3 \\
\hline
\end{tabular}

Fuente: Instituto Nacional de Estadística e informática -Encuesta Permanente de Empleo

Sin embargo, la pobreza, como lo indica Reglero (2014), no es el factor principal para definir las oportunidades de las personas y la posibilidad de poder relacionarse y desarrollarse dentro del entorno en el que viven; factores exógenos a estos son los que los han llevado a esta situación; entender la pobreza y la exclusión social también abarca o incluye a la privación de la ciudadanía. Subirats (2004) -citado en el artículo de Reglero- explica "que no se puede seguir hablando de 
pobreza como una razón de las desigualdades sociales porque, aunque es evidente que las carencias económicas continúan siendo un factor determinante para analizarlas, no pueden tratarse como únicas, ya que nuevos mecanismos y factores de segregación están actuando a la hora de impedir la plena integración de las personas en el ámbito laboral, formativo o cultural. Paradójicamente, en la actualidad, el retroceso en la consecución de derechos sociales genera la vuelta de la utilización del término pobreza referido casi exclusivamente a necesidades básicas".

Uno de los conceptos relacionados con la pobreza es el de la privación, que va de la mano con los de subsistencia y las necesidades básicas. Se define como el estado de desventaja relativa a la comunidad local, sociedad o la nación a la que pertenece el individuo, la familia o el grupo. Sobre este concepto, podemos describir un sinnúmero de limitaciones tanto sociales como materiales que los restringen, y con los cuales podemos categorizar como causa los que con mayor énfasis atacan a determinados grupos sociales (Benach y Amable, 2004).

\section{Causa-efecto de una pandemia social}

Hablar de la pobreza generada alrededor de las ciudades como un problema social es como no querer definir claramente qué la ocasiona, de dónde parten los problemas y por dónde deben comenzar las soluciones. El efecto de migración parte por la búsqueda de posibilidades de subsistencia; empieza por el abandono y el olvido del Estado de la población que vive en su territorio y no es correctamente atendida, por una falta de evaluación de las posibilidades de desarrollo en cada zona habitada del país y también de una falta de planificación para sustentar las mismas.

En Andrade y Jiménez-Bandala (2017), que cita a Myrdal (1957), se explica la teoría de la "causación circular acumulativa"; ella indica que los países con limitaciones económicas e índices altos de pobreza padecen de un "efecto de acumulación cíclico", lo que hace que las brechas entre países pobres y ricos se amplíen. Esto es determinado porque factores independientes como la pobreza pueden generar que los gobiernos inviertan menos en educación y que esta situación origine 
un bajo índice en el nivel competitivo futuro de la población (variable dependiente). El efecto circular condicionaría a que la educación en el futuro se convierta en una variable independiente, dado que la falta de nivel educativo no permitirá fácilmente acceso al empleo.

\section{A. Empleo informal}

Bajo estos términos, la posibilidad de la generación de empleo informal se hace más latente. Por ejemplo, Freije (2002), en un estudio desarrollado para el Banco Interamericano de Desarrollo (BID), indica que el sector informal se relaciona con la excesiva regulación vigente por parte del Estado, pero también existen factores ligados a la falta de educación y preparación en las competencias que requiere el mercado laboral para poder incorporarse a un trabajo formal. La formalización requiere de pago de impuestos elevados y regulaciones burocráticas, que hacen costosa o impiden a las empresas pequeñas ser rentables, sumado a la imposibilidad de acceder al crédito.

En la búsqueda por hacerse de ingresos que permitan sostener necesidades básicas, el empleo informal pasa a ser la única posibilidad que tiene la población de escasas posibilidades para integrarse a una sociedad de consumo con acceso al crédito. Perez-Roa (2019), en su artículo "Emprendedores por necesidad...", resalta lo difícil que puede ser para un grupo de la sociedad chilena con limitaciones económicas sostener el pago de un crédito. El problema se torna complejo para las parejas jóvenes por lo que tendrán que buscar otra fuente de ingreso; la primera podrá ser formal y temporal, y concretar un segundo empleo, y la segunda se guiará hacia un empleo informal asociado a la venta de productos o trabajos esporádicos en ese sector.

El sector informal hace referencia al conjunto de unidades productivas no constituidas en sociedad del sector institucional de los hogares, que no se encuentran registradas en la administración tributaria (INEI, 2017). Indica que el empleo informal concierne aproximadamente a tres de cada cuatro peruanos de la Población Económicamente Activa Ocupada (población ocupada). Señala también que "la informalidad es producto de la incapacidad del sector 
moderno de la economía capitalista para absorber el gran contingente de mano de obra generada en el mercado de trabajo. De esta forma, un gran segmento de mano de obra desocupada tiene como única salida la autogeneración de empleos de subsistencia de baja productividad".

La economía informal se fomenta por la falta de capacidad por parte del Estado de promover el trabajo formal mejorando las condiciones de fomento del empleo, las inversiones y la calidad de la educación, por lo que los grupos menos favorecidos estarán fuera de la economía formal (Puntes-León AJ. et al., 2016). Él señala, tomando en cuenta lo indicado por la OIT, que "aunque algunas actividades ofrecen medios de vida e ingresos razonables, la mayoría de las personas que trabajan en la economía informal están expuestas a condiciones de trabajo inadecuadas e inseguras y tienen altos niveles de analfabetismo, poca calificación y escasas oportunidades de formación; tienen ingresos más inciertos, más irregulares y más bajos, que quienes trabajan en la economía formal, laboran más horas, no gozan de derechos de negociación colectiva ni de representación y a menudo, su situación de empleo es ambigua o encubierta; tienen una mayor vulnerabilidad física y financiera porque el trabajo en la economía informal está excluido o efectivamente fuera del alcance de los regímenes de seguridad social y de la legislación en materia de protección de la seguridad y la salud, la maternidad y otras normas de protección de los trabajadores".

Las precarias condiciones y jornadas de trabajo, sumadas a las remuneraciones bajas y al riesgo laboral pueden establecer una relación muy alta con la salud de los trabajadores y su jornada de trabajo, que, en muchos aspectos, al no encontrarse regulada, ocupa gran parte del tiempo del trabajador, que se puede extender a 16 horas en un solo día. La exposición de su salud y el riesgo de posibles accidentes a que se encuentran sometidos los trabajadores informales podrían ser de orden físico, químico o biológico. Es importante tener en cuenta que este tipo de trabajo no cuenta con ninguna relación de apoyo jurídico y del Estado, ya que se encuentra a merced del empleador (Bernandino y Andrade, 2015).

La búsqueda de la calidad de vida ofrece como resultado migraciones de la población a las ciudades; refiriéndonos específicamente a esta 
búsqueda, podemos determinar que, finalmente, la calidad de vida es una aspiración de todo ser humano, por lo tanto, el acceso al trabajo permite buscar la propia autonomía, poder enlazarla a las propias decisiones, a poseer libertad, así como ser un medio para la realización personal y socialización. Sin embargo, la precariedad y la incapacidad para generar ingresos produce un impacto en la salud y afecta también al componente psicológico (Vélez et al., 2015).

\section{B. Rol del Estado}

Otra mirada de la relación que buscamos como causa-efecto de la informalidad, la vulnerabilidad y la marginalidad se encuentra en el Estado. Teniendo en cuenta que la informalidad afecta directamente a la productividad y la recaudación tributaria, factores que hemos mencionado, como el exceso de regulación tributaria, el pago de beneficios sociales y otros, limitan la búsqueda de la formalización y el fomento del empleo. Pasa la factura el hecho de la poca productividad que pueden mostrar los potenciales trabajadores, debido a la falta de capacidades técnicas asociadas a la educación. Otro aspecto es que la oferta del Estado para la formalización, llámese servicios básicos y de seguridad, no es atractiva (Belapatińo et al., 2017).

El Estado cuenta con algunas armas en situaciones de crecimiento que son paliativos para el logro de la reducción de la pobreza, como son los ingresos fiscales, el canon, las regalías mineras y petroleras. Sin embargo, estos sistemas quedan vulnerados cuando el crecimiento se reduce o por situaciones como las que se viven actualmente con la pandemia de la COVID-19; el crecimiento pasa a índices negativos; en este caso, la población en general se va a ver afectada y no estará dispuesta a aceptar mayor presión tributaria o de asistencia (Herrera, 2017).

Es el Estado el que influye directamente en la formalización del empleo, en las regulaciones de este y en las leyes que lo fomentan o que lo limitan, debido a que promueve el desarrollo urbano no planificado, sin analizar las consecuencias que genera, maneja el sustento de los servicios básicos que tanto el sector informal como el formal utilizan. Tan solo el lado formal es el que lo sostiene; por lo tanto, es limitada 
la recaudación de impuestos. Con respecto a las políticas fiscales y al manejo económico, el Estado está más preocupado de manejar las cifras de inflación y crecimiento, y menos dispuesto a la generación del empleo en el manejo de políticas macroeconómicas (Freije, 2002).

\section{Factores psicosociales}

En Ruiz (2012), vemos que los factores que miden la vulnerabilidad desde el punto de vista psicosocial deben contener parámetros con los que se puedan evaluar la satisfacción del significado por ejemplo de bienestar, vida digna, salud, vivienda adecuada, etc.

Sólo así los satisfactores pueden convertirse en un estándar normativo que pueda tomarse como punto de referencia para evaluar el bienestar, o para medir la intensidad de las condiciones de vulnerabilidad y los efectos dañinos y/o degradantes de una situación específica de crisis para un individuo, hogar o grupo social. Sin estos elementos, enfrentar las situaciones de vulnerabilidad se reduce a 'un retorno a la normalidad', cuando en realidad implica mucho más que eso; requiere una visión de la vulnerabilidad como las condiciones que generan una afectación mayor a la vida tanto de individuos como colectividades y, por lo tanto, un problema de ética social.

Si bien es necesario medir los parámetros que definen la situación de vulnerabilidad, es importante tener en cuenta las características psicológicas de las poblaciones vulnerables, y esto nos lleva analizar a los diferentes grupos que son afectados y su poca posibilidad de generar una respuesta a los riesgos que se presentan en aspectos de seguridad y cuya exposición que se hace latente en los niños, las niñas y las mujeres. La integración familiar se hace más inestable, frente a las condiciones de privación y precariedad. "El riesgo, es decir, la probabilidad e intensidad de que la merma en el bienestar tenga lugar puede cuantificarse, pero difícilmente medirá el impacto de la incertidumbre en la vida de quien está expuesto a la amenaza" (Aristegui et al., 2017). 
La amenaza que existe con respecto a la vulnerabilidad de los nińos y las nińas que se desarrollan en ambientes de carencias económicas, de vivienda y salud, en los que los padres carecen de instrucción adecuada, existe hacinamiento y falta de espacios para una dinámica familiar, las posibilidades de riesgo y la generación de "transformaciones estructurales traumáticas que pueden ser tan adversos como los desastres socio-naturales en tanto implican cambios drásticos en las condiciones de vida de las personas, familias y comunidades". El problema que existe es que no contar con recursos para manejar la situación, "[...] se traduce en la incapacidad de afrontamiento o la disminución de las posibilidades y oportunidades de minimizar estos impactos" (Estevez, 2011).

Desde el punto de vista social, la vulnerabilidad se ha tratado de diferentes formas y ha abordado diferentes aspectos. La Declaración universal sobre bioética y derechos humanos, en 2005, analiza los aspectos de protección y derechos de los individuos integrados a la sociedad, que debe expresar un análisis ético en cuanto a la dignidad con la que deben vivir las personas y, en esta línea, el sentido de la solidaridad también cobra un aspecto importante. La fragilidad de las personas vulnerables y las posibilidades de desarrollarse y sustentarse, sumadas a "[...] las condiciones desfavorables que exponen a las personas a mayores riesgos, a situaciones de falta de poder o control, a la imposibilidad de cambiar sus circunstancias" (Feito, 2007), debe movernos a la acción y a un cambio de actitud.

Las consecuencias de la precariedad económica vulneran profundamente los hogares de las familias que se encuentran en estas condiciones. En muchos aspectos, estudios realizados en este ámbito como los de Ortiz-Ruiz y Díaz-Grajales (2018) en el municipio de Cali, Colombia, donde la familia y las condiciones de bajos recursos fueron el aspecto resaltante, "se encontraron relacionados con el manejo de los conflictos o deterioro de las relaciones familiares, así como los asociados con la relación problemática entre capital humano (salud-educación) y trabajo/empleo. Del mismo modo, se evidencia una relación de desventaja entre ingresos, necesidades, dinámicas familiares y gastos. Por último, aun cuando se señaló que la vivienda es uno de los principales 
activos, las posibilidades para acceder a vivienda propia son escasas". Encuentran relaciones conflictivas con respecto a cuestiones económicas, al agrandamiento de las familias por un factor de embarazo de las niñas o las adolescentes con el consiguiente aumento de los gastos familiares, la violencia familiar, el empleo precario por la falta de una buena educación, la discapacidad relacionada con la edad de los adultos mayores.

\section{Salud y salubridad}

Existe una relación entre la pobreza, la salud y la desnutrición. Esta relación nos permite observar cómo las poblaciones de bajos recursos económicos viven en limitadas condiciones de salud, prueba de ello está en las capacidades sanitarias en las que se desarrollan y las instalaciones de los centros de salud con las que cuentan. La falta de educación de la familia en aspectos sanitarios influye en las enfermedades, lo mismo que las condiciones de sus viviendas y las zonas en las que viven, las cuales no cuentan muchas veces con los servicios básicos de agua y electricidad. Todo ello incide en el nivel de mortalidad de adultos y niños que son los más expuestos a enfermedades intestinales y respiratorias.

Wagstaff (2002) nos ofrece un panorama similar en el análisis de la pobreza y su relación con la salud; indica que "los pobres tienden a morir antes y a presentar mayores niveles de morbilidad que quienes tienen más dinero". Indica que existe una correlación entre la mala salud con la malnutrición y la mortalidad; asimismo, que los niños de familias de bajos recursos de los países en desarrollo tienen una menor probabilidad de estar vacunados pese a las campańas nacionales de vacunación. Esto se debe en parte a la incapacidad de los servicios de salud para llegar a los pobres. Indica también que son limitantes la capacidad de instalaciones y la capacidad del personal de atención de los centros de salud, teniendo en cuenta la distancia a la que se encuentran los mismos.

Uno de los problemas que aqueja a Latinoamérica es la mala reputación que ostenta "de ser la región en el mundo con mayor desigualdad social. Existe una amplia brecha, que sigue ensanchándose, entre ricos y pobres; y el criterio democrático liberal [...] que se 
viene aplicando a la organización social favorece tal ampliación. De resultas de ello, hay en el continente grandes diferencias en materia de resultados de salud entre grupos sociales. Aunque la estratificación social es manifiesta en las diferencias de salud de todas las sociedades y de todos los estratos de la sociedad, parece que las brechas son mayores en América que en otros lugares. Se suelen atribuir las diferencias sanitarias a esta desigualdad social, y varios estudios revelan que tales diferencias entre los países podrían atribuirse en parte a la mala distribución de los ingresos" (Alleyne, 2002).

\section{Desnutrición infantil}

Según el Centro Nacional de Alimentación y Nutrición del Ministerio de Salud, el Perú presentaba un $16 \%$ de desnutrición crónica en los niños menores de 5 años en el año 2019. "La desnutrición en la niñez menor de cinco años incrementa su riesgo de muerte, inhibe su desarrollo cognitivo y afecta a su estado de salud de por vida. Atender a este problema es condición indispensable para asegurar el derecho a la supervivencia y al desarrollo de las niñas y niños de América Latina y el Caribe, así como para garantizar el desarrollo de los países" (Martínez y Fernández, 2006). Se indica que la mitad de los problemas de nutrición ocurren en las zonas rurales con exposición a riesgos ambientales, donde no existen instalaciones de agua potable y saneamiento. Otro aspecto importante es que la desnutrición se relaciona con la extrema pobreza, los bajos ingresos, el bajo nivel educativo de los padres, la falta de atención primaria de la salud (instalaciones deficientes o inexistentes). El impacto que se genera en esta situación está dado en la "morbimortalidad, educación y productividad, constituyéndose en uno de los principales mecanismos de transmisión intergeneracional de la pobreza y la desigualdad".

\section{E. Riesgos sociales de la pobreza y vulnerabilidad}

Uno de los problemas sociales que enfrentamos con respecto a la vulnerabilidad y a la pobreza se puede manifestar en los niños, las niñas y los y las adolescentes. Rubio (2017) hace un análisis muy claro sobre 
este tema; indica que los problemas por los que pasa este grupo social son de "naturaleza dinámica y multidimensional y van acompañados de aspectos como los de la salud, riesgos naturales, discriminación o violencia”. Rubio indica que los afecta en el desarrollo todo su potencial, el cual quedaría limitado. "Los niños y niñas atraviesan complejos procesos de desarrollo físico, psicológico e intelectual y tienden a ser más vulnerables que los adultos ante la pobreza, la desnutrición, las enfermedades, el abuso y la explotación".

La trascendencia de los problemas sociales que se dan frente a las limitaciones económicas de los hogares puede acarrear la posibilidad de la presencia de conductas de riesgo como el abuso de las drogas, la delincuencia y el abandono escolar. Se puede afirmar que estas condiciones no necesariamente pueden tener un común denominador en la pobreza; sin embargo, sí tienen una relación en cuanto al desarrollo, a las aspiraciones y al futuro (proyecto de vida), que desencadenaría en la frustración personal (Deza, 2015).

Analizando el desarrollo de los niños, las niñas, los adolescentes y los jóvenes, los riesgos expuestos y la proyección de estos grupos humanos a la sociedad a través de la ciudadanía, nos obliga a pensar en los derechos civiles, políticos y sociales, con los que se han formado; "[...] también entran en juego el concepto de justicia social, el principio de legalidad y las obligaciones de los ciudadanos". Si estas condiciones no se dan en su formación y proyección, la desigualdad en el ámbito social que genera estará marcada por una brecha en su desarrollo humano, social y económico, que finalmente perjudicará el futuro del país (Castillo, 2016).

\section{Conclusiones}

Cuando habla de la "urbanización de la pobreza", Foschiatti (2007) se refiere al progresivo avance de la pobreza a través de las migraciones de poblaciones sin recursos en búsqueda de mejorar sus condiciones de vida. De Soto (2001) incide en la precariedad de las viviendas que construyen e indica que al final no van a pertenecerles, debido a que el Estado, que permite las invasiones, no genera la propiedad individual 
de las zonas invadidas. La falta de empleo que activa en estos grupos la economía informal debido al exceso de normas y leyes por parte del Estado finalmente termina marginando a los participantes de las ventajas de la legalidad (Sandoval y Loayza, 2014).

El desempleo y la educación nos llevan a la marginalización de la persona en la sociedad; aunque Navarro y Larrubia (2006) indican que hay mucho campo y mucha discusión para definir el marco de la marginalidad; sin embargo, se puede analizar derivando ciertos parámetros y elementos que lo contengan. Por lo que Carrillo et al. (2019), tomando en cuenta la educación como parte de esta variable de marginación, parte de la premisa que una mala educación genera menores oportunidades de aprendizaje, por los bajos niveles de los centros educativos a los que acuden los niños, las niñas y los y las adolescentes en condiciones de pobreza (Cuenca y Urrutia, 2019).

Las condiciones de pobreza limitan la participación de los padres en las labores de apoyo en la educación de sus hijos (Castro y Cano, 2013); estas limitantes, sumadas a la precaria educación que reciben, determinarán su capacidad económica, sustento y desarrollo social para el futuro; estos problemas se agravan con la suma de la mala alimentación, salud física y mental recibida en sus hogares (Castro $e t$ al., 2017), (Stock et al., 2014).

Sobre el futuro y el desarrollo social, Reglero (2014) indica que los factores de pobreza abarcan e incluyen la privación de la ciudadanía; categorizar las causas que generan las limitaciones sociales que nos privan y nos marginan son indicadores que debemos tomar en cuenta para analizar cuáles son los más pertinentes en determinados grupos sociales (Benach y Amable, 2004).

En este ámbito, es importante analizar la informalidad a través del empleo; las poblaciones con bajo nivel educativo, pocas posibilidades de integrarse a un trabajo formal y sin acceso al crédito, terminan buscando en el empleo informal o en la informalidad una posibilidad de sustento (Perez-Roa, 2019). Según cifras del INEI (2017), tres de cada cuatro peruanos se encuentran empleados informalmente. Uno de los factores de este problema está en la incapacidad del Estado de promover el empleo formal (Puntes-León et al., 2016), tampoco de 
analizar o controlar los perjuicios salariales, condiciones de trabajo, exposición de salud y riesgo de accidentes que genera la informalidad (Bernandino y Andrade, 2015).

El rol del Estado en la formación integral de las capacidades técnicas de su población juega un papel decisivo en la formalización y el futuro de la productividad de un país (Belapatiño et al., 2017), y que son paliativos para la reducción de la pobreza (Herrera, 2017). En las políticas de gobierno, se ha dado más énfasis en las cifras y en los indicadores macroeconómicos que en la generación del empleo formal (Freije, 2002).

En este ámbito, el Estado da la espalda a problemas que generan una formación de personas vulnerables. Estas finalmente incrementarán las cifras de pobreza, por lo que el factor psicosocial, desde el punto de vista de la vulnerabilidad, les impedirá generar respuestas adecuadas a los riesgos en los que viven. Finalmente, este grupo social no encontrará sustento en el significado de bienestar, vida digna, salud y vivienda adecuada (Ruiz, 2012), (Aristegui et al., 2017). Es latente esta amenaza en los niños y las niñas que se desarrollan en estos ambientes de precariedad con significativas carencias económicas y de salud; en el futuro marcará la capacidad de ellos para poder afrontar posteriormente y con mayor certeza soluciones en su desarrollo y crecimiento personal. Se le suman a esta situación los conflictos familiares, el deterioro de sus relaciones, riesgos de embarazo y violencia familiar, entre otros (Feito, 2007), (Estevez, 2011), (Ortiz-Ruiz y Díaz-Grajales, 2018).

La desnutrición infantil y la pobreza se unen a un factor que compete al Estado. Sin embargo, este tiene una limitada actuación en cuestión de salud pública y las políticas que se deben desarrollar a partir de la búsqueda de soluciones concretas, debido a que la población en extrema pobreza tiene propensión a reducir sus expectativas de vida. El rol del Estado en estas circunstancias se ve limitado por una falta de planificación y programación su capacidad de atención y prevención en los menos privilegiados económicamente hablando (Wagstaff, 2002), (Alleyne, 2002).

Otro factor incremental ligado a la vulnerabilidad es la nutrición infantil; el efecto que causa en la población infantil una mala nutrición 
es el de incrementar su riesgo de muerte, inhibir su desarrollo cognitivo y afectar su estado de salud de por vida (Martínez y Fernández, 2006). Se suman a esto la desnutrición, las enfermedades, el abuso y la explotación; esta situación puede acarrear como consecuencia la posibilidad de la presencia de conductas de riesgo, como el abuso de las drogas, la delincuencia, el abandono escolar (Deza, 2015), (Rubio, 2017).

El futuro ciudadano de los niños y las niñas que forman parte de nuestra sociedad, ante situaciones adversas, puede crear una brecha en su desarrollo humano, social y económico (Castillo, 2016). El Estado, que debe formar parte de la solución, se encuentra limitado muchas veces por la generación de trasfondos políticos y partidarios, la corrupción y la crisis política; resolver los problemas de fondo requiere más que un actor y las soluciones a los problemas endémicos de una sociedad deben ser asumidos por todos.

Las cifras y los análisis nos dan un punto de partida para bosquejar el problema con el fin de desarrollar y planificar soluciones desde el análisis académico, político, de la sociedad civil y la empresa privada. El futuro de un país se encuentra en el crecimiento y el desarrollo del tesoro más grande al que debemos proteger y atender: la niñez y la juventud. Lo que hagamos hoy será la semilla que determinará si los cimientos y el dinamismo con el que sembramos nos darán una cosecha abundante, próspera y sostenible. 


\section{Bibliografía:}

Alleyne George (2002). La equidad y la meta de Salud para todos. Rev Panam Salud Publica/Pan Am J Public Health 11(5/6), 2002.

Aristegui, Iratxe; Beloki, Usue; Díez, Ainhoa y Silvestre, María (2017). "Vulnerabilidad social percibida en contexto de crisis económica». Revista Española de Sociología (RES), 26 (3), 17-39.

Belapatińo V., Grippa F. \& Perea H. (2017). Perú | Informalidad laboral y algunas propuestas para reducirla. Observatorio Económico Perú. BBVA Research.

Beltrán, A., \& Seinfeild, J. (2014). Desnutrición Crónica Infantil en el Perú: Un problema persistente. Lima: Universidad del Pacífico.

Benach, Joan, \& Amable, Marcelo. (2004). Las clases sociales y la pobreza. Gaceta Sanitaria, 18(Supl. 1), 16-23. Recuperado de: http://scielo.isciii.es/scielo.php?script=sci_arttext\&pid=S0213$91112004000400005 \& \operatorname{lng}=$ es\&tlng=es.

Bernardino, Débora Cristina de Almeida Mariano, \& Andrade, Marilda. (2015). O Trabalho Informal e as Repercussōes para a Saúde do Trabalhador: Uma Revisão Integrativa. Revista de Enfermagem Referência, serIV(7), 149-158. https://dx.doi. org/10.12707/RIV14049

Carrillo, S., Salazar, V. \& Sayuri, L. (2019). Jóvenes y educación en Lima Metropolitana y Callao / Sandra Carrillo, Víctor Salazar y Sayuri Leandro Lima; IEP, 2019 (Documento de Trabajo, 259. Educación 19)

Castillo Pérez, N. (2016). Desarrollo humano, desigualdad y pobreza. Cultura De Paz, 22(68), 10-19. https://doi.org/10.5377/ cultura.v22i68.2719

Castro Salinas, Rodolfo, Rivera, Renzo, \& Seperak, Rosa. (2017). Impacto de composición familiar en los niveles de pobreza de Perú. Cultura-hombre-sociedad, 27(2), pp. 69-88. https://dx.doi. org/10.7770/cuhso-v27n2-art1229 
Castro, L \& Cano, R. (2013). Pobreza y vulnerabilidad: Factores de riesgo en el proceso educativo. Contextos educativos 16, 55 - 72.

Córdoba, A (2020), Pandemia, informalidad y tributación. Diario Gestión: https://gestion.pe/opinion/pandemia-informalidad-ytributacion-noticia/11/06/2020

Cuenca, R. \& Urrutia C. (2019). Explorando las bhas de la desigualdad educativa en el Perú. RMIE, 2019, Vol.24, Núm. 81, PP. 431461 (ISSN: 14056666).

De Soto, Hernando (2001). "El Misterio del Capital". Ed. Ediciones Península 1ra edición.

Deza S. (2015). Factores de riesgo y protección en nińos y adolescentes en situación de pobreza de Instituciones Educativas en Villa El Salvador. Av.psicol. 23(2) 2015240 agosto-diciembre. DOI: https://doi.org/10.40.00/poldev.2518

Estevez, Alicia (2011). Vulnerabilidad psicosocial: una aproximación conceptual. III Congreso Internacional de Investigación y Práctica Profesional en Psicología XviII Jornadas de Investigación Séptimo Encuentro de Investigadores en Psicología del MERCOSUR. Facultad de Psicología - Universidad de Buenos Aires, Buenos Aires.

Feito, L. (2007). Vulnerabilidad. Anales del Sistema Sanitario de Navarra, 30(Supl. 3), 07-22. Recuperado de: http:// scielo.isciii.es/scielo.php?script=sci_arttext\&pid=S113766272007000600002\&lng=es\&tlng=es.

Foschiatti, A. M. ${ }^{a}$ H. (2007). Vulnerabilidad, pobreza y exclusión. Problemas de gran impacto en la población del nordeste argentino. Anales de Geografía ISSN: 0211-9803. 2007, vol. 27, núm. 2, 9-40.

Freije, S. (2002). El empleo informal en América Latina y el Caribe: causas, consecuencias y recomendaciones de política 
(Documentos de Trabajo Mercado Laboral, BID). Washington: BID. Recuperado de: http://idbdocs.iadb.org/wsdocs/ getdocument.aspx?docnum $=354774$

Herrera J. (2017). "Pobreza y desigualdad económica en el Perú durante el boom de crecimiento: 2004-2014", International Development Policy Revue Internationale de Politique de Développement [Online], 9 | 2017, Online since 04 April 2018, connection on 31 October 2020. Recuperado de: <http:// journals.openedition.org/poldev/2518>.

INEI (2017). "Producción y Empleo Informal en el Perú, Cuenta Satélite de la Economía Informal 2007-2017”. Instituto Nacional de Estadística e Informática.

INEI, (2020). Fuente permanente de empleo. Recuperado de: https:// www.inei.gob.pe/media/MenuRecursivo/boletines/10-informetecnico-mercado-laboral-jul-ago-set-2020.pdf

Instituto Nacional de Salud (2020). Sala Situacional Estado Nutricional Niños menores de 5 años SIEN-HIS 2019. Ministerio de Salud del Perú. Recuperado de: https://web.ins.gob.pe/es/ alimentacion-y-nutricion/vigilancia-alimentaria-y-nutricional/ sala-nutricional

Loayza N. (2008). Causas y consecuencias de la informalidad en el Perú. BCR Revista Estudios Económicos, 2008. Recuperado de: <https://www.bcrp.gob.pe/docs/Publicaciones/RevistaEstudios-Economicos/15/Estudios-Economicos-15-3.pdf>.

Martínez, Rodrigo y Andrés Fernández (2006). Desnutrición infantil en América Latina y el Caribe. Número 2, abril de 2006, Publicación de las Naciones Unidas, abril de 2006. ISSN versión impresa 1816-7527, ISSN versión electrónica 1816-7535.

Matos Mar, José (1984). Desborde popular y crisis del Estado. El nuevo rostro del Perú en la década de 1980. Lima: Instituto de Estudios Peruanos. 
Myrdal, G. (1957). Economic Theory Underdevelopet Region. London: Gerald Duckworth \& Co.

Navarro, R. y Larrubia, R. (2006). Indicadores para medir situaciones de vulnerabilidad social. Propuesta realizada en el marco de un proyecto europeo. Baética: Estudios de arte, geografía e historia.2006; 28(1): p. 485-506.

Ortiz-Ruiz, Nicolás y Constanza Díaz Grajales (2018). Una mirada a la vulnerabilidad social desde las familias. Revista Mexicana de Sociología 80(3), 611-638. https://doi.org/10.22201/ iis.01882503p.2018.3.57739

Pérez-Roa, L. (2019). "Emprendedores por necesidad: el emprendimiento como estrategia de pago de deudas en un contexto precariedad laboral". Aposta. Revista de Ciencias Sociales, 83, pp. 61-75. Recuperado de: http://apostadigital. $\mathrm{com} /$ revistav3/hemeroteca/loperez.pdf

Puentes-León KJ., Rincón-Bayona LY. y Puentes-Suárez A. Análisis bibliométrico sobre trabajo y salud laboral en trabajadores informales, 2010-2016. Rev. Fac. Nac. Salud Pública. 2018;36(3):71-89.

Reglero, M. (2014). Utilización de los términos pobreza y exclusión: Una cuestión dinámica. Educación y Futuro, 30 (2014), pp. 3959. ISSN: $1576-5199$.

Roca Rey, I. y Rojas, B (2002). "Pobreza y exclusión social: una aproximación al caso peruano", Bulletin de l'Institut français d'études andines [en línea], 31 (3), 2002. Recuperado de: http://journals.openedition.org/bifea/6828; DOI : 10.4000/ bifea. 6828

Rodríguez, A. I. (2011). Problemas en torno a la definición de la marginalidad. Trabajos y Comunicaciones (37), 203-219. En Memoria Académica. 
Rosas, L., Y Jiménez-Bandala, C. (2018). El desempleo y la probabilidad de caer en trampas de pobreza - Unemployment and the Probability of Falling into Poverty Traps: Consideraciones para países en vías de desarrollo. Reis: Revista Española De Investigaciones Sociológicas, (164), 3-20. doi:10.2307/26633315

Rubio, M. (2017). La construcción de un sistema de protección social adecuado a la infancia en el Perú, Oportunidades y retos. Publicación de las Naciones Unidas (CEPAL), LC/TS.2017/144.

Ruiz Rivera, Naxhelli. (2012). La definición y medición de la vulnerabilidad social. Un enfoque normativo. Investigaciones geográficas, (77), pp. 63-74. Recuperado de: http://www. scielo.org.mx/scielo.php?script=sci_arttext\&pid=S018846112012000100006\&lng=es\&tlng=es.

Sandoval, G. (2014). La informalidad laboral: causas generales. Equidad y Desarrollo, (22), pp. 9-45. https://doi.org/10.19052/ed.3247

Stock, L., Corlyon J., Castellanos C. \& Gieve M. (2014). Personal Relationships and Poverty an Evidence and Policy Review. Londres: The Tavistock Institute. Recuperado de: http://bit. ly/2C6jlvs.

Subirats, J. (2005). Análisis de los factores de exclusión social (Documentos de trabajo, 4). Bilbao: fundación BBVA.

Vélez, C., Pico, M. E. y M. P. Escobar (2015). Determinantes sociales de la salud y calidad de vida en trabajadores informales. Manizales, Colombia. Salud de los Trabajadores, ISSN-e 1315-0138, Vol. 23, No. 2, 2015, pp. 95-100.

Wagstaff Adam (2002). Pobreza y desigualdades en el sector de la salud. Revista Panamericana de Salud Pública, Volumen 11, Numero 5-6. 